\title{
Changes in Matrix Metalloproteinase and Tissue Inhibitors of Metalloproteinase in Patients with Rotator Cuff Tears
}

\author{
Oh-Soo Kwon, Young-Yul Kim, Ji Yoon Ha, Han Bit Kang \\ Department of Orthopedic Surgery, The Catholic University of Korea, Daejeon St. Mary's Hospital, Daejeon, Korea
}

Background: The purpose of this study was to determine whether in patients with rotator cuff tears a correlation exists between molecular changes and clinical parameters such as age, duration of symptom, range of motion, and tear size. Molecular changes of matrix metalloproteinase (MMP) and tissue inhibitor of metalloproteinase (TIMP) were assessed by measuring messenger RNA (mRNA) levels of the two proteins.

Methods: The rotator cuff tissue from was obtained from the edge of a torn tendon revealed after debridement by a motorized shaver. Using the sample of rotator cuff tissue, the reverse transcription polymerase chain reaction was performed to quantify MMP-2 and TIMP2 mRNA expression. To determine whether mRNA levels and the clinical variables, such as age, defect size, range of motion (ROM) of shoulder, and duration of symptoms, show any correlation, Spearman's correlation coefficients were used to test for significant differences.

Results: There was an inverse correlation between the mRNA levels of MMP-2 and TIMP-2 from the torn rotator cuff tendons regardless of the clinical variables. However, comparison of mRNA levels versus clinical parameters such as age, defect size, range of motion and duration of symptoms revealed a number of findings. We found a significant correlation between age and mRNA levels of MMP-2 from torn cuffs $(r=0.513, p=0.021)$. Further, we found a significant correlation between defect size in the full thickness tears and mRNA levels of MMP-2 ( $r=0.454, p=0.045)$. Conversely, no significant association between mRNA levels of MMP-2 and ROM or duration of symptom was found.

Conclusions: Our results suggest that both MMP-2 and TIMP-2 may be involved in the disease process of rotator cuff tears. Although the level of mRNA expression of MMP-2 and TMP-2 remain constant in torn rotator cuffs irrespective of the clinical variables, their levels may be influenced by age and defect size, which could account to change in tendon degradation and the healing process.

(Clin Shoulder Elb 2014;17(2):64-67)

Key Words: Rotator cuff; Matrix metalloproteinase; Tissue inhibitor of metalloproteinase, Polymerase chain reaction

\section{Introduction}

Rotator cuff tears are common cause of pain in the shoulder joints. ${ }^{1)}$ The rotator cuff is responsible for the dynamics stability of the shoulder joint and receives considerable stress during shoulder exercises. Rotator cuff tears manifest as pain in the shoulder joint that cause suboptimal function. The debilitating changes in rotator cuffs range from being a reversible tendinitis to forming a tear across all levels of the rotator cuff. Natural healing of rotator cuff tears without surgical intervention is difficult, which is why open or arthroscopic repairs have been implemented and have been shown to give clinically successful outcomes. ${ }^{2-4)}$ Despite a high rate of clinical success in the repairs in rotator cuff, studies that look at the biomolecular or histological patterns in the degenerative changes such as decrease in cell count, disorganization of fibrous tissue, formation of granulation tissue, glycosaminoglycan infiltration, changes in fibrocartilage, and calcification were noted that would eventually lead to

Received February 25, 2014. Revised March 20, 2014. Accepted April 30, 2014.

Correspondence to: Oh-Soo Kwon

Department of Orthopedic Surgery, The Catholic University of Korea, Daejeon St. Mary's Hospital, 64 Daeheung-ro, Jung-gu, Daejeon 301-723, Korea

Tel: +82-42-220-9248, Fax: +82-42-221-4120, E-mail: oskn@catholic.ac.kr

Financial support: None. Conflict of interests: None. 
complete tears. However, studies that look into the molecular mechanisms behind these changes are sparse.

Matrix metalloproteinase (MMP) is a biological enzyme that mediate breakdown of polypeptides, of which around 20 types are known so far. MMP is a zinc dependent endopeptidase that is essential to break down extracellular matrix components. The ability of MMP to regulate the extracellular medium contributes to local infiltration, vascularization, metastasis of tumor cells etc. MMP is classified according to the type of substrates they target. For example, MMP-1, -8, -13 are primary collagenases, and MMP-3, $-10,-11$ are streptolysins that target a broader range of substrates. The endogenous inhibitor of MMP is tissue inhibitor matrix metalloproteinase (TIMP). As an inhibitor that can inhibit the action of all known types of MMPs, any defects in TIMP expression, formation, and recycling, may lead to degenerative conditions such as rheumatoid arthritis. Other molecular functions of TIMPs exist, but in rotator cuff tears, the concentration of TIMP-3 has been apoptosis. ${ }^{5,6)}$

Of these, MMP-2 is an enzyme that can break down substances such as gelatin at a fast rate, and its activity is essential for collagen breakdown in soft tissues. Animal studies that had used shoulder muscles to study the regenerative process have found increased expression of MMP-2 during the recovery period of the rotator cuff. Further, an increase in MMP-13 and IL-1 $\beta$ was proposed as a possible biomarker of an imminent rotator cuff tear. ${ }^{6,7)}$ As such, this study aimed to look whether the expression of MMP and the expression of TIMP, the enzyme that inhibits MMP expression, are associated with clinical variables, such as age, duration of symptom, range of motion, and tear size in patients with rotator cuff tears.

Table 1. The Sequence of Primers Used in the Experiment

\begin{tabular}{ll}
\hline \multicolumn{1}{c}{ Primer } & \multicolumn{1}{c}{ Sequences } \\
\hline $\begin{array}{l}\text { MMP-2 (199 bp) } \\
\text { Forward }\end{array}$ & 5'-GGCCCTGTCACTCCGAGAT-3' \\
Reverse & 5'-GGCATCCAGGTTATCGGGGA-3' \\
TIMP-2 (400 bp) & \\
Forward & 5'-GGCGTTTTGCAATGCAGATGTAG-3' \\
Reverse & 5'-CACAGGAGCCGTCACTTCTCTTG-3' \\
TIMP-1 (400 bp) & \\
Forward & 5'-GCGGATCCAGCGCCCAGAGAGACAC-3' \\
Reverse & 5'-TTAAGCTTCCACTCCGGGCAGGATT-3' \\
MMP-9 (247 bp) & \\
Forward & 5'-CACTGTCCACCCCTCAGAGC-3' \\
Reverse & 5'-GCCACTTGTCGGCGATAAGG-3' \\
GAPDH (200 bp) & \\
Forward & 5'-TAAAGGGCATCCGGGCTACACT-3' \\
Reverse & 5'-TTACTCCTTGGAGGCCATGTAGG-3' \\
\hline
\end{tabular}

MMP: matrix metalloproteinase, TIMP: tissue inhibitor of metalloproteinase, GAPDH: glyceraldehyde 3-phosphate dehydrogenase.

\section{Methods}

Tissues were obtained from each patient once consent was given and all consent forms were filled out by the patient. All procedures relating to human tissue sampling were approved by the human ethics review committee of The Catholic University of Korea, Daejeon St. Mary's Hospital. Of the 20 rotator cuff tear patients, 13 had complete tears and 7 had partial tears. The tissues were obtained during the arthroscopic repair. The relative ratio of male to female was 12:8. The mean age of patients was 59 years (range, $39-76$ years), and the average duration of symptoms was 10 months (range, 3-24 months). Further, for patients with complete rotator cuff tears a probe with $1 \mathrm{~mm}$ increments was used to measure the defect size, and the average defect size was measured as $439 \mathrm{~mm}^{2}$ (range, $150-600 \mathrm{~mm}^{2}$ ).

The tissue were collected during arthroscopic debridement and stored in a $-70^{\circ} \mathrm{C}$ freezer. For the quantification of messenger RNA (mRNA), primers against MMP-2, TIMP-2, MMP9, and TIMP-1 were created (See Table 1 for primer sequences) and the reverse transcription polymerase chain reaction (RTPCR) was performed. In brief, total RNA was extracted from the tissue using the Trizol kit (Invitrogen, Carlsbad, CA, USA). Then, complementary DNA (CDNA) was produced using the extracted RNA ( $2 \mu \mathrm{g})$ as template by reverse transcription using the Superscript system from Invitrogen. The conditions for RT-PCR were as follows; denaturation step for $3 \mathrm{~min}$ at $94^{\circ} \mathrm{C}$, primer annealing step for $1 \mathrm{~min}$ at $65^{\circ} \mathrm{C}$, and extension step for $1 \mathrm{~min}$ at $72^{\circ} \mathrm{C}$. The number of PCR cycle used was 35 cycles. To check for PCR products, electrophoresis at $70 \mathrm{~V}$ for $90 \mathrm{~min}$ was performed on all products on $1.5 \%$ agarose gels. The relative density of each band was measured using the Image J program $(\mathrm{NIH}$, Bethesda, MD, USA).

Spearman correlation coefficients were calculated by SPSS program ver. 12.0 (SPSS Inc., Chicago, IL, USA) to determine correlation between the clinical variables (age, defect size, range of motion [ROM] of shoulder, and duration of symptoms) and

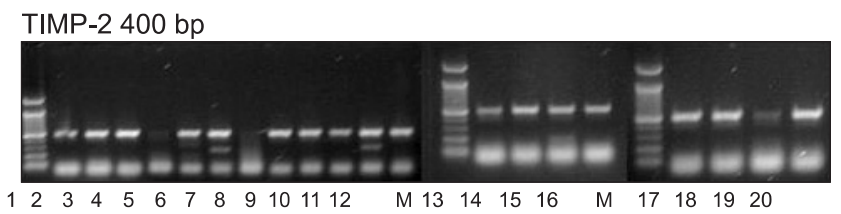

MMP-2 $370 \mathrm{bp}$

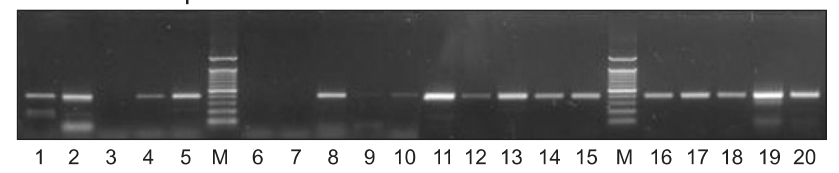

Fig. 1. Electrophoresis of polymerase chain reaction products show bands at sizes $\sim 370$ bp and $\sim 400$ bp that represent expression of MMP-2 and TIMP-2, respectively. MMP: matrix metalloproteinase, TIMP: tissue inhibitor of metalloproteinase, M: marker. 

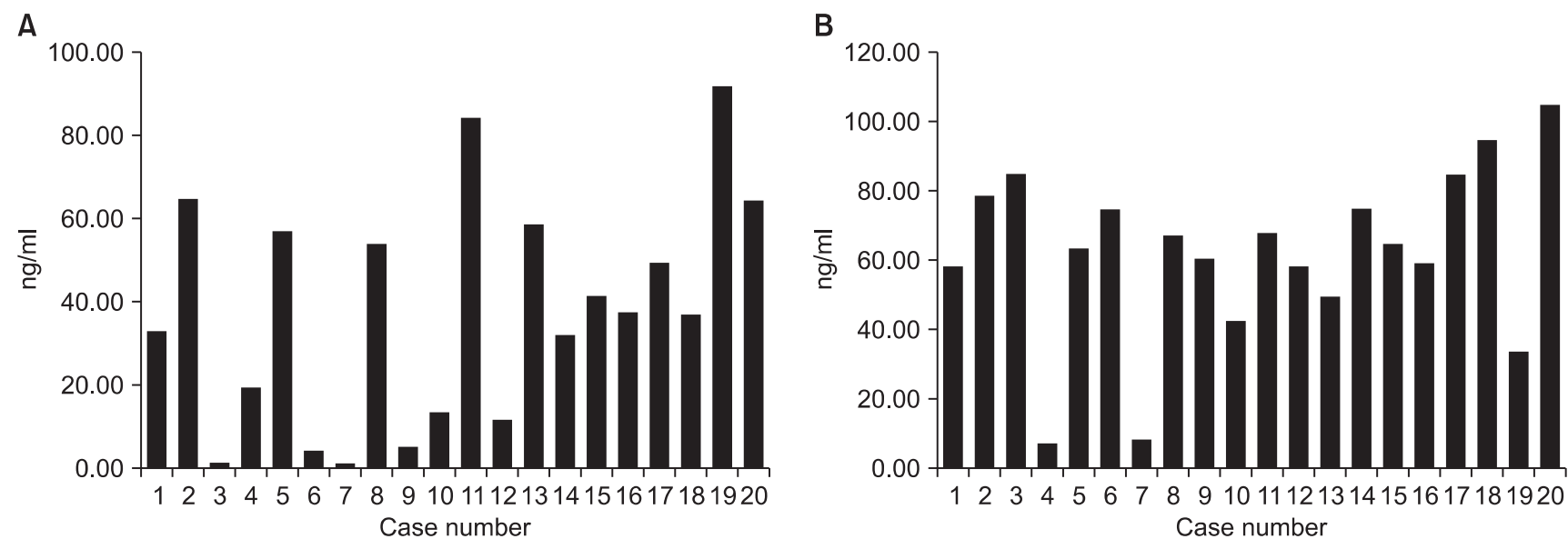

Fig. 2. An inverse correlation between MMP-2 (A) and TIMP-2 (B) in torn rotator cuffs. MMP: matrix metalloproteinase, TIMP: tissue inhibitor of metalloproteinase.
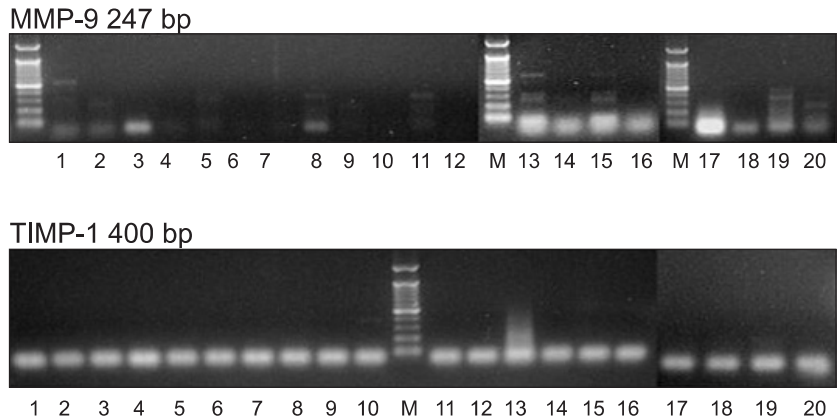

Fig. 3. Expression of MMP-9 and TIMP-1 demonstrates a less remarkable pattern compared to MMP-2. MMP: matrix metalloproteinase, TIMP: tissue inhibitor of metalloproteinase, M: marker.

mRNA levels. The level of significance was set at $p<0.05$.

\section{Results}

We found that the MMP-2 and TIMP-2 were expressed in all patients irrespective of the clinical variables, and that the mRNA levels of MMP-2 and TIMP-2 were inversely proportional (Fig. 1, 2). The mRNA transcripts of MMP-9 and TIMP-1 were not expressed in most samples, and similarly the mRNA level of TIMP2 was expressed in very low amounts (Fig. 3). A correlation analysis of the expression level of the two mRNA transcripts with age, defect size, ROM and duration of symptoms showed that, the expression of MMP-2 mRNA is significantly correlated to age and defect size $(r=0.513, p=0.021)$, but not ROM or duration of symptoms (Table 2).

\section{Discussion}

MMP is important for tissue regeneration, and even though the exact mechanism is unknown, the overexpression of MMP
Table 2. Correlation between mRNA Levels and Clinical Variables

\begin{tabular}{lc}
\hline \multicolumn{1}{c}{ Variable } & Correlation coefficient $^{*}$ \\
\hline Age & 0.513 \\
Duration of symptoms & -0.613 \\
Tear size & 0.454 \\
Range of motion (forward flexion) & 0.009 \\
\hline
\end{tabular}

${ }^{*}$ By Spearman's correlation.

in muscle during tissue regeneration is thought to elicit lesions, especially under stress-induced conditions. ${ }^{8)}$ Conversely, TIMP is involved in counteracting stress conditions. Further, MMP modulates expression of collagen and other extracellular matrix components important for the rotator cuff tear recovery process. ${ }^{8)}$ Previous report has shown that in response to acute lesions, the rotator cuffs respond by switching the gene expression of type 1 collagen to type 3 collagen, and MMP has been shown to be involved in such regenerative processes. ${ }^{9,10)}$

MMP requires zinc as a cofactor for its enzymatic activity. MMP is known to be involved in both normal and pathophysiological processes, such as degeneration or regeneration, involving proteolysis and peptide formation inside the tissue and in the extracellular matrix space. For example, MMP-2 is a key factor required for the efficient breaks down collagen. Imbalance of collagen reconstruction is attributed to changes in MMP and TIMP levels. A decrease in MMP-3 and stromelysin-1, increase and/or decrease in MMP-2 (gelatinase $A$ ), and changes in mRNA levels of MMP-1, -9 and -13 TIMP 2-4 was seen in some studies. A study using rabbit showed that MMP-2 and TIMP-1 are expressed in both damaged and regenerated tissue, thereby highlighting their possible role in recovery of a damaged supraspinatous. Similarly, in a different study, changes in mRNA concentration of MMP-13 also indicated the possible role of these 
enzymes in the recovery process. ${ }^{10-12)}$

Select studies have shown that in degeneration or in tendon lesions, the mRNA expression level of MMP is inversely correlated to that of TIMP. However, Choi et al. ${ }^{13)}$ reported that MMP2 expression is not only seen in tendons of surgically excised tissue but also in regenerated tissue. Other studies, ${ }^{14,15)}$ have also shown increased expression of MMP-2 and MMP-9 in synovium tissue. Such studies have assessed mRNA expression, but did not analyze a possible relationship between mRNA levels and clinical variables, as in our study. Lakemeier et al. ${ }^{11)}$ found that whilst the position and size of the rotator cuff tear influences that biology of MMPs, the concentration of MMP-1 and MMP-9 at the articular surface of a partial tear is significantly greater than at the surface of the synovium. Prospective studies that similarly assess changes in the clinical variables that are correlated with changes in the biomarkers of rotator cuff tears. Factors that inhibit MMP have been assessed previously, but results had shown that drug or mechanical induction of changes in MMP was found to cause a varied influence on recovery.

Limitations to this studies came from only obtaining a small sample volume, not being able to obtain healthy tissue for ethical reasons, thus any correlations we could make could not be compared in the context of an undamaged tissue.

\section{Conclusion}

Therefore, our results show that despite a constant expression of MMP-2 and TIMP-1 in damaged tissue, their expression is unrelated to any clinical variable we tested. Our findings show that the mRNA levels of MMP-2 and TIMP-1 may be influenced by age and defect size. Thus, prospective studies into how changes in mRNA levels of MMP-2 and TIMP-1 may contribute to the pathophysiology of rotator cuff tears should be addressed, if any.

\section{References}

1. Murrell GA, Walton JR. Diagnosis of rotator cuff tears. Lancet. 2001;357(9258):769-70.

2. Cofield RH, Parvizi J, Hoffmeyer PJ, Lanzer WL, Ilstrup DM, Rowland CM. Surgical repair of chronic rotator cuff tears. A prospective long-term study. J Bone Joint Surg Am. 2001;83$\mathrm{A}(1): 71-7$.

3. Hattrup SJ. Rotator cuff repair: relevance of patient age. J Shoulder Elbow Surg. 1995;4(2):95-100.

4. Jensen KL, Williams GR Jr, Russell IJ, Rockwood CA Jr. Rotator cuff tear arthropathy. J Bone Joint Surg Am. 1999;81(9):131224.

5. Tetlow LC, Adlam DJ, Woolley DE. Matrix metalloproteinase and proinflammatory cytokine production by chondrocytes of human osteoarthritic cartilage: associations with degenerative changes. Arthritis Rheum. 2001;44(3):585-94.

6. Yoshihara Y, Nakamura H, Obata K, et al. Matrix metalloproteinases and tissue inhibitors of metalloproteinases in synovial fluids from patients with rheumatoid arthritis or osteoarthritis. Ann Rheum Dis. 2000;59(6):455-61.

7. Dalton S, Cawston TE, Riley GP, Bayley IJ, Hazleman BL. Human shoulder tendon biopsy samples in organ culture produce procollagenase and tissue inhibitor of metalloproteinases. Ann Rheum Dis. 1995;54(7):571-7.

8. Bedi A, Kovacevic D, Hettrich C, et al. The effect of matrix metalloproteinase inhibition on tendon-to-bone healing in a rotator cuff repair model. J Shoulder Elbow Surg. 2010;19(3):38491.

9. Lo IK, Marchuk LL, Hollinshead R, Hart DA, Frank CB. Matrix metalloproteinase and tissue inhibitor of matrix metalloproteinase mRNA levels are specifically altered in torn rotator cuff tendons. Am J Sports Med. 2004;32(5):1223-9.

10. Riley GP, Curry V, DeGroot J, et al. Matrix metalloproteinase activities and their relationship with collagen remodelling in tendon pathology. Matrix Biol. 2002;21(2):185-95.

11. Lakemeier S, Braun J, Efe T, et al. Expression of matrix metalloproteinases 1, 3, and 9 in differing extents of tendon retraction in the torn rotator cuff. Knee Surg Sports Traumatol Arthrosc. 2011;19(10):1760-5.

12. Thornton GM, Shao X, Chung M, et al. Changes in mechanical loading lead to tendonspecific alterations in MMP and TIMP expression: influence of stress deprivation and intermittent cyclic hydrostatic compression on rat supraspinatus and Achilles tendons. Br J Sports Med. 2010;44(10):698-703.

13. Choi HR, Kondo S, Hirose K, Ishiguro N, Hasegawa Y, Iwata $\mathrm{H}$. Expression and enzymatic activity of MMP-2 during healing process of the acute supraspinatus tendon tear in rabbits. J Orthop Res. 2002;20(5):927-33.

14. Ritty TM, Herzog J. Tendon cells produce gelatinases in response to type I collagen attachment. J Orthop Res. 2003;21(3):442-50.

15. Castagna A, Cesari E, Gigante A, Conti M, Garofalo R. Metalloproteases and their inhibitors are altered in both torn and intact rotator cuff tendons. Musculoskelet Surg. 2013;97 Suppl $1: 39-47$. 